\title{
Going Past the Data for Temozolomide
}

J. Lee Villano, M.D., Ph.D., Nathalie Letarte, B.Pharm, M.Sc, Linda R. Bressler, Pharm.

D.

Departments of Medicine (JLV), Neurosurgery (JLV) and Pharmacy Practice (LRB)

University of Illinois at Chicago, Chicago, Illinois

Faculty of Pharmacy, Universite de Montreal, Montreal, Canada (NL)

Corresponding Author: $\quad$ J. Lee Villano, M.D., Ph.D.

Associate Professor of Medicine and Neurosurgery

Director of Medical Neuro-Oncology

Section of Hematology/Oncology

840 S. Wood St. Rm. 839 (M/C 713)

Chicago IL 60612

Tele: 312-996-6768

Fax: (312) 413-4205

e-mail: jvillano@uic.edu 


\section{Temozolomide}

\section{ABSTRACT (if required):}

The benefit of six cycles of adjuvant temozolomide was documented in a randomized phase III (EORTC-NCIC CE.3) trial and this therapy, following combined temozolomide and radiation, is the standard of care for patients with newly diagnosed glioblastoma. We comment on the differences on length of adjuvant therapy in both clinical practice and national studies (e.g. RTOG 0825), usually doubling the length than in the EORTC/NCIC study, and relate to historic adjuvant trials for solid tumors.

BODY:

The benefit of six cycles of adjuvant temozolomide was documented in a randomized phase III (EORTC-NCIC CE.3) trial and this therapy, following combined temozolomide and radiation, has become the standard of care for patients with newly diagnosed glioblastoma (GBM). ${ }^{1}$ Forty per cent of patients in this phase III trial did not complete the planned six cycles of adjuvant temozolomide. The most common reason noted for patients receiving less than six cycles was disease progression. Following the acceptance of this therapy as the standard of care, it is apparently not uncommon to continue temozolomide for longer than six cycles sometimes for an arbitrary number of cycles, and sometimes until disease progression. The feasibility and tolerability of such long-term therapy has been documented in case series and single institution reports in both the recurrent and adjuvant settings. ${ }^{2-8}$ However, the benefit of long-term therapy cannot be documented in these uncontrolled experiences - patients have to live long enough 
Temozolomide

or live long enough without progression in order to receive more cycles. The rationale for administering more cycles, especially in the adjuvant setting, is not clear. It does not appear to be extent of disease resection - gross complete resection versus subtotal resection. In a phase II study evaluating survival at 16 months following informed consent, bevacizumab was added to combined temozolomide and radiation, and bevacizumab and irinotecan were added to adjuvant temozolomide. ${ }^{4}$ The initial study treatment plan specified six cycles of adjuvant therapy. The treatment plan was later changed to allow up to twelve cycles of adjuvant treatment, in response to patients' hesitancy or unwillingness to discontinue after six cycles. A landmark analysis of those who received six versus those who received more than six cycles demonstrated no difference in progressionfree or overall survival between these two groups. ${ }^{4}$ Two subsequent, ongoing phase III trials, the primary objectives of which are overall survival, with the addition of bevacizumab to standard adjuvant temozolomide, and with dose intensive versus standard dose adjuvant temozolomide, respectively, specify six cycles in all treatment arms, with the option to continue to a maximum of twelve cycles, provided there is evidence of continued benefit.9,10 This option for up to twelve cycles of maintenance temozolomide has also been widely applied to investigation of the Stupp regimen in lower grade gliomas: Grade II: E3F0511 and Grade III: NCCTG-N0577 and RTOG-0834.12,13 This arbitrary doubling of standard duration of treatment would be unacceptable for many adjuvant trials for solid tumors, and likely challenges statistical interpretation. 
Temozolomide

The response to patients' desires to continue for more than six cycles is

understandable. But we would argue that the benefit of more than six cycles has not been proven in a randomized controlled trial. Further, there is a small but documented risk of serious hematologic adverse events with temozolomide, including aplastic anemia ${ }^{14,15}$, myelodysplastic syndrome ${ }^{14,16}$ and treatment-related acute myeloid leukemia. ${ }^{14,17}$ The risk/benefit ratio could change with deviations from the well studied regimen, and it may not be in the best interest of patients to continue beyond six cycles, in the absence of documented benefit.

It is also possible that less than six cycles of adjuvant therapy could prove to be as effective as the standard six cycles. In stage IIIB non-small cell lung cancer, the results of a phase II study led to the frequent use of three cycles of consolidation docetaxel after completion of two cycles of cisplatin and etoposide with concurrent chest radiation therapy. ${ }^{18}$ However, when the role of docetaxel consolidation was formally evaluated in a randomized phase III trial, no benefit was seen, and patients in the consolidation arm experienced greater toxicity. ${ }^{19}$

In recent months, there has been considerable discussion and debate following reports of second primary malignancies in patients receiving long -term lenalidomide for multiple myeloma, leading to the premature termination of lenalidomide maintenance in the French IFM 2005-02 trial. ${ }^{20}$ It is true that time to progression and survival time in myeloma are generally longer than those in GBM, thus patients with GBM may not live long enough to develop treatment-related 
complications. But without a documented benefit, is it appropriate to expose patients to any increased risk?

In today's world of exciting research to discover molecular markers that predict response or toxicity, and manipulation of treatments to prevent or overcome resistance, a randomized comparison of standard versus prolonged or less than standard adjuvant temozolomide may seem somewhat boring. But studies designed to answer similar questions in breast cancer ${ }^{21}$, testicular cancer ${ }^{22}$ and colon cancer $^{23}$ have led to improved outcomes or improved tolerability, or both. Acknowledgement: We would like to thank ALLIANCE Neuro-oncology Committee members and Roger Stupp for discussion of these issues.

\section{References:}

1. Stupp R, Hegi ME, Mason WP, et al: Effects of radiotherapy with concomitant and adjuvant temozolomide versus radiotherapy alone on survival in glioblastoma in a randomised phase III study: 5-year analysis of the EORTCNCIC trial. Lancet Oncol 10:459-66, 2009

2. Brandes AA, Franceschi E, Tosoni A, et al: MGMT promoter methylation status can predict the incidence and outcome of pseudoprogression after concomitant radiochemotherapy in newly diagnosed glioblastoma patients. J Clin Oncol 26:2192-7, 2008

3. Lai A, Filka E, McGibbon B, et al: Phase II pilot study of bevacizumab in combination with temozolomide and regional radiation therapy for up-front treatment of patients with newly diagnosed glioblastoma multiforme: interim analysis of safety and tolerability. Int J Radiat Oncol Biol Phys 71:1372-80, 2008

4. Vredenburgh JJ, Desjardins A, Reardon DA, et al: The addition of bevacizumab to standard radiation therapy and temozolomide followed by bevacizumab, temozolomide, and irinotecan for newly diagnosed glioblastoma. Clin Cancer Res 17:4119-24, 2011

5. Hau P, Koch D, Hundsberger T, et al: Safety and feasibility of long-term temozolomide treatment in patients with high-grade glioma. Neurology 68:688-90, 2007 
6. Khasraw M, Bell D, Wheeler H: Long-term use of temozolomide: could you use temozolomide safely for life in gliomas? J Clin Neurosci 16:854-5, 2009

7. Poelen J, Prick MJ, Jeuken JW, et al: Six year survival after prolonged temozolomide treatment in a 30-year-old patient with glioblastoma. Acta Neurol Belg 109:238-42, 2009

8. Stern JI, Apisarnthanarax S, Paleologos NA, et al: Temozolomide as long-term Maintenance treatment for gliomas. Annals of Neurology 54:S31, 2003

9. Gilbert M, Wang M, Aldape K, et al: RTOG 0525: A randomized phase III trial comparing standard adjuvant temozolomide (TMZ) with a dose-dense (dd) schedule in newly diagnosed glioblastoma (GBM). J Clin Oncol 29:suppl; abstr 2006, 2011

10. National Cancer Institute: Temozolomide and Radiation Therapy With or Without Bevacizumab in Treating Patients With Newly Diagnosed Glioblastoma, In: ClinicalTrials.gov. Bethesda (MD): National Library of Medicine (US). Available from: http://clinicaltrials.gov/show/NCT00626990 NLM Identifier: NCT00626990, 2011

11. National Cancer Institute: Radiation Therapy With or Without Temozolomide in Treating Patients With Low-Grade Glioma, In: ClinicalTrials.gov. Bethesda (MD): National Library of Medicine (US). Available from: http://clinicaltrials.gov/show/NCT00978458 NLM Identifier: NCT00978458, 2011

12. National Cancer Institute: Radiation Therapy With or Without Temozolomide in Treating Patients With Anaplastic Glioma, In: ClinicalTrials.gov. Bethesda (MD): National Library of Medicine (US). Available from: http://clinicaltrials.gov/show/NCT00626990 NLM Identifier: NCT00626990, 2011

13. National Cancer Institute: Radiation Therapy or Radiation Therapy and Temozolomide or Temozolomide Alone in Treating Patients With Newly Diagnosed Anaplastic Glioma, In: ClinicalTrials.gov. Bethesda (MD): National Library of Medicine (US). Available from: http://clinicaltrials.gov/show/NCT00887146 NLM Identifier: NCT00887146, 2011

14. Villano JL, Letarte N, Yu JM, et al: Hematologic adverse events associated with temozolomide. Cancer Chemother Pharmacol, 2011

15. FDA: Temozolomide (marketed as Temodar): Aplastic Anemia. Drug safety newsletter 1:7, 2007

16. Chamberlain MC, Raizer J: Extended exposure to alkylator chemotherapy: delayed appearance of myelodysplasia. J Neurooncol 93:229-32, 2009

17. De Vita S, De Matteis S, Laurenti L, et al: Secondary Ph+ acute lymphoblastic leukemia after temozolomide. Ann Hematol 84:760-2, 2005

18. Gandara DR, Chansky K, Albain KS, et al: Consolidation docetaxel after concurrent chemoradiotherapy in stage IIIB non-small-cell lung cancer: phase II Southwest Oncology Group Study S9504. J Clin Oncol 21:2004-10, 2003

19. Hanna N, Neubauer M, Yiannoutsos C, et al: Phase III study of cisplatin, etoposide, and concurrent chest radiation with or without consolidation 
docetaxel in patients with inoperable stage III non-small-cell lung cancer: the Hoosier Oncology Group and U.S. Oncology. J Clin Oncol 26:5755-60, 2008

20. The Myeloma Beacon Staff: Revlimid Cancer Controversy Flares, URL: http://www.myelomabeacon.com/news/2011/02/05/revlimid-lenalidomide-cancercontroversy-flares/. 2011

21. Tancini G, Bonadonna G, Valagussa P, et al: Adjuvant CMF in breast cancer: comparative 5-year results of 12 versus 6 cycles. J Clin Oncol 1:2-10, 1983

22. Einhorn LH, Williams SD, Troner M, et al: The role of maintenance therapy in disseminated testicular cancer. N Engl J Med 305:727-31, 1981

23. O'Connell MJ, Laurie JA, Kahn M, et al: Prospectively randomized trial of postoperative adjuvant chemotherapy in patients with high-risk colon cancer. J Clin Oncol 16:295-300, 1998 